\title{
Capacitor Current Feedback Active Damping with Lagged Compensator for DFIG Wind Turbines with $L C L$ Filter
}

\author{
Leyre Rosado, Javier Samanes, Eugenio Gubía and Jesús López \\ Electrical, Electronic and Communication Engineering \\ Institute of Smart Cities \\ Public University of Navarre (UPNA) \\ Pamplona, Spain \\ leyre.rosado@unavarra.es
}

\begin{abstract}
Several active damping strategies have been proposed in the literature for grid-connected converters with $L C L$ filter but there are not specific strategies for DFIG wind turbines. In this system, there is an interaction between the two converters of the back-to-back conversion structure, which must be properly modeled in order to design effective damping strategies for the $L C L$ filter resonant poles. This paper proposes a robust active damping strategy for DFIG wind turbines with $L C L$ filter that considers the special features of this system. In this technique the filter capacitor current is fed back through a lag compensator that adjusts the delay of the feedback loop to emulate a virtual impedance that has dominant resistive behavior in the range of possible resonance frequencies. It is shown that a similar damping of the $L C L$ filter resonance is achieved when the strategy is implemented in either of the two converters.

Index Terms-Doubly-fed induction generator (DFIG) wind turbines, $L C L$ filter resonance, active damping, stability analysis.
\end{abstract}

\section{INTRODUCTION}

Wind energy conversion systems with doubly-fed induction generators (DFIG) are a very common configuration in onshore wind turbines, given the cost reduction of using a back-to-back power converter that only works with a third of the generated power [1]. $L C L$ filters are a standard solution to filter high-frequency harmonics caused by the pulse-width modulation that also reduce the system cost compared to $L$ filters [2]. However, they introduce stability problems since the $L C L$ filter resonance interacts with the converter control. Therefore, a damping technique is required to solve this problem, preferably an active damping (AD) strategy since they do not add any extra cost [3].

AD strategies can be classified in two main groups: single loop and multiloop strategies. Single loop AD strategies introduce a digital filter in the current control loop, normally a low pass filter, a notch filter or a lead-lag compensator [4], [5]. They do not require additional sensors but they may be more sensitive to variations in the grid inductance, since it modifies

This work was supported by the Spanish State Research Agency (AEI) under grants PID2019-110956RB-I00 /AEI/ 10.13039 and DPI-2016-80641R.

( 2020 IEEE. Personal use of this material is permitted. Permission from IEEE must be obtained for all other uses, in any current or future media, including reprinting/ republishing this material for advertising or promotional purposes, creating new collective works, for resale or redistribution to servers or lists, or reuse of any copyrighted component of this work in other work. the resonance frequency. The value of the grid inductance depends on the grid short circuit ratio (SCR), which can lead to a wide range of possible resonance frequencies.

Multiloop AD strategies feed back an additional variable to ensure stability [6]. The most common multiloop $\mathrm{AD}$ is the filter capacitor current proportional feedback. Due to the control delays, this method is equivalent to a virtual impedance in parallel with the filter capacitor, which reduces the stability region to $f_{s} / 6$, where $f_{s}$ is the sampling frequency [7]. To extend this region, some techniques have been proposed such as reducing the computation delay [7]. In [8] the critical frequency is extended to $0.45 f_{s}$ by introducing a recursive infinite impulse response digital filter in the feedback path. [9] and [10] manage to extend the stability range up to almost the Nyquist frequency using a quasi-integral element and a PI function in the feedback path, respectively. However, the range of possible resonance frequencies is bounded by the SCR limits at the point of common coupling (PCC), so it can be more interesting to maximize the damping in this range, rather than extending the stability region as much as possible. This is done by emulating a virtual impedance with high resistance and low reactance in the resonance frequency range.

All the previous AD strategies are developed for a gridconnected voltage source converter, however, there are several characteristics in DFIG wind turbines that have to be considered to adjust AD strategies. From Fig. 1, it can be derived that the grid side converter (GSC) and the rotor side converter (RSC) are connected in parallel to the grid, thus their dynamics are coupled. Besides, the impedance of the DFIG machine interacts with the impedance of the $L C L$ filter, modifying the resonance frequency range [11]. This interaction must be considered to design effective AD strategies for DFIG wind turbines.

In this paper a multiloop AD strategy is proposed. The capacitor current is fed back through a lag compensator, which is tuned to emulate a virtual impedance with dominant resistance component in the range of possible resonance frequencies, so that the damping is maximized in this range and the strategy is robust against grid inductance variations. The $\mathrm{AD}$ design 


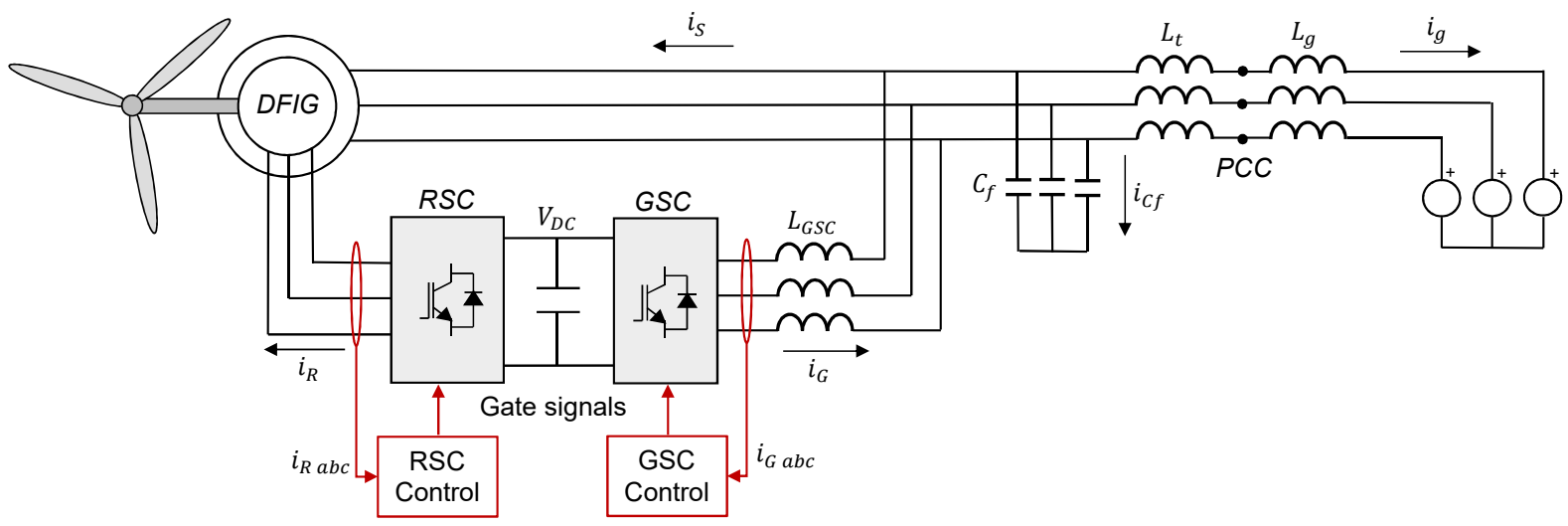

Fig. 1. DFIG wind turbine with $L C L$ filter connected to the grid.

takes into consideration the special characteristics of the DFIG wind turbine. For that purpose, a complete system model is developed that includes the interaction between the GSC and the RSC control loops, as well as the interaction between the $L C L$ filter and the DFIG impedances. Finally, it is shown that the system can be damped from either of the two converters, obtaining a similar damping in the system resonant poles.

\section{System Modeling And STABILITy AnALYSiS}

The system under study is a DFIG wind turbine as the one represented in Fig. 1. In this configuration, the stator is directly connected to the grid, whereas a back-to-back power converter is connected to the rotor windings. It is formed by two converters, the grid side converter and the rotor side converter. The RSC controls the torque and the rotational speed of the generator, whereas the GSC controls the DC-bus voltage. The GSC is connected to the stator through the inductor $L_{G S C}$, which is the converter side inductor of the harmonic $L C L$ filter. The filter capacitor is $C_{f}$ and the grid side inductor is formed by $L_{t}$ and $L_{g} . L_{t}$ is the step up transformer leakage inductance that connects the DFIG system to the medium voltage grid at the point of common coupling, and $L_{g}$ is the grid inductance. The value of $L_{g}$ depends on the grid short circuit ratio (SCR), which is a measure of the grid strength. The GSC and the RSC are controlled as current sources using a PI controller, as represented in the block diagram of Fig. 2. Both converters act on the same plant, which is formed by the DFIG machine and the $L C L$ filter, proving that there is an interaction between both control loops. The AD feedback, in red, is explained in detail in the next section.

\section{A. Plant Model}

All the elements must be modeled in the same reference frame in order to analyze the system stability. Normally, DFIG wind turbines are controlled in the synchronous reference frame, $d q$, so all the components are modeled in this reference frame. As a result, cross-coupling terms appear between the variables in both axis. Therefore, all the components are modeled using $2 \times 2$ impedance matrices, which are defined between brackets in this paper. The angular speed of the crosscoupling terms is indicated as a subscript outside the brackets. Vectors are defined in capital letters with the subscript $d q$ to denote that they are expressed in the synchronous reference frame.

1) Harmonic LCL Filter Model: The dynamics of the $L C L$ filter can be described by three equations. The grid voltage is neglected because it does not influence the stability of the system since it is a disturbance in the current control loop.

$$
\begin{gathered}
I_{G d q}=\left[Z_{R L_{G S C}}\right]_{\omega_{0}}^{-1}\left(V_{G d q}-V_{C f d q}\right) \\
I_{g d q}=\left[Z_{R L_{g}}\right]_{\omega_{0}}^{-1} V_{C f d q} \\
V_{C f d q}=\left[Z_{C f}\right]_{\omega_{0}}\left(I_{G d q}-I_{g d q}-I_{S d q}\right) .
\end{gathered}
$$

$V_{G d q}$ and $I_{G d q}$ are the GSC output voltage and current, $V_{C f d q}$ is the filter capacitor voltage, $I_{g d q}$ is the grid current and $I_{S d q}$ is the stator current. $\left[Z_{R L_{G S C}}\right] \omega_{0},\left[Z_{R L_{g}}\right] \omega_{0}$ and $\left[Z_{C f}\right]_{\omega_{0}}$ are the impedance matrices of the components of the $L C L$ filter. The inductors are modeled with a series resistance and an inductance. For the converter side inductor, $\left[Z_{R L_{G S C}}\right]_{\omega_{0}}$, $R_{G S C}$ is the series resistance and $L_{G S C}$ is the inductance. In the case of the grid side inductor, $\left[Z_{R L_{g}}\right]_{\omega_{0}}$, the series resistance is $R_{g}$ and the inductance is $L_{t}+L_{g}$. The filter capacitor impedance matrix, $\left[Z_{C f}\right]_{\omega_{0}}$, includes the capacitance $C_{f} . \omega_{0}$ is the angular speed of the $d q$ axis, which is equal to the grid fundamental speed.

2) DFIG Model: In the model of the DFIG generator, the subscripts $S$ and $R$ denote whether the voltage and the current belong to the stator or rotor terminals, respectively. The stator and rotor voltages, $V_{S d q}$ and $V_{R d q}$, are expressed as a function of the currents, $I_{S d q}$ and $I_{R d q}$, as follows

$$
V_{S d q}=\left[Z_{L_{m}}\right] \omega_{0} I_{R d q}+\left[Z_{R L_{S}}\right]_{\omega_{0}} I_{S d q}
$$




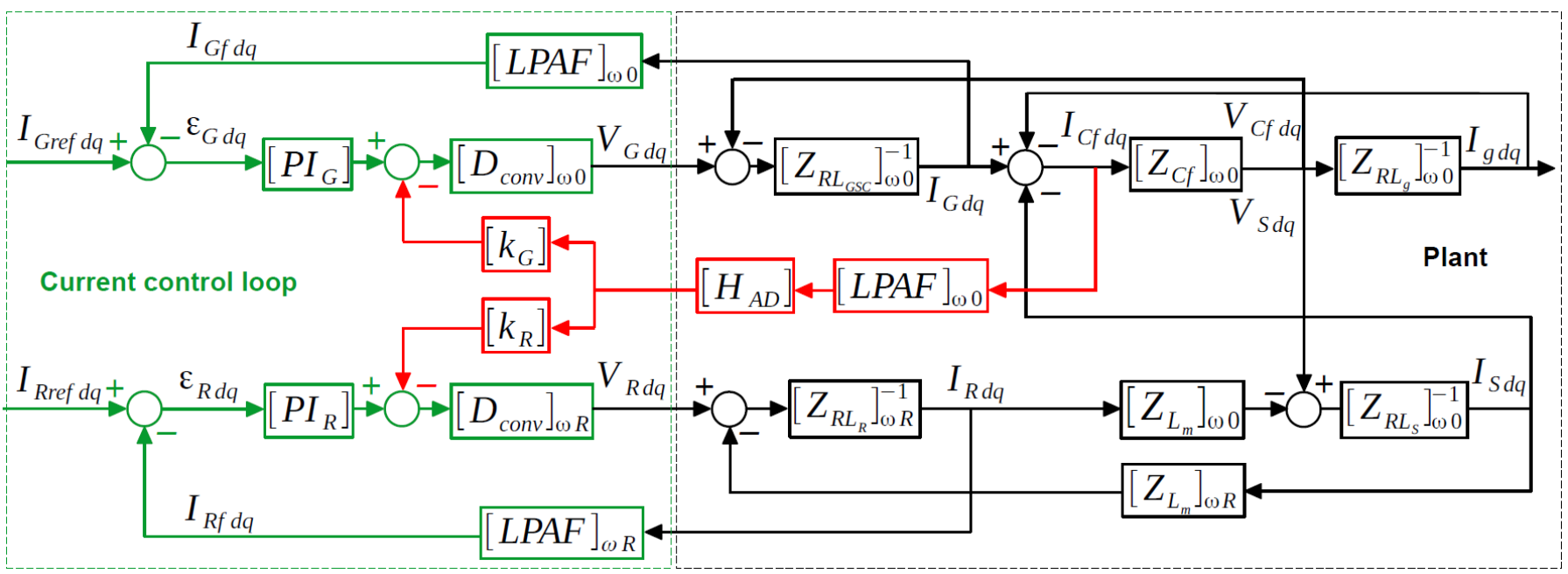

Fig. 2. Block diagram of the GSC and RSC current control loops.

$$
V_{R d q}=\left[Z_{R L_{R}}\right]_{\omega_{R}} I_{R d q}+\left[Z_{L_{m}}\right]_{\omega_{R}} I_{S d q} .
$$

$\left[Z_{L_{m}}\right]_{\omega}$ is the impedance matrix that models the magnetizing inductance, $L_{m}$. Note that the angular speed of the cross-coupling terms, $\omega$, is different in (4) and (5). $\omega_{R}$ is the difference between $\omega_{0}$ and the rotor electrical angular speed, $\omega_{m} .\left[Z_{R L_{S}}\right]_{\omega_{0}}$ models the stator total inductance, $L_{S}$, and the stator windings series resistance, $R_{S}$. $L_{S}$ equals the sum of $L_{m}$ and the stator leakage inductance. Similarly, $\left[Z_{R L_{R}}\right]_{\omega_{R}}$ models the rotor total inductance, $L_{R}$, and its series resistance, $R_{R} . L_{R}$ also equals the sum of $L_{m}$ and the rotor leakage inductance. All the parameters and variables are referred to the stator.

Finally, the stator voltage equals the filter capacitor voltage

$$
V_{S d q}=V_{C f d q} .
$$

\section{B. GSC and RSC Control Loop Model}

The GSC and the RSC currents are filtered by a low pass analog filter, $\operatorname{LPAF}(s)=1 /(\tau s+1)$. $D_{\text {conv }}(s)$ models the delays within the control loop. There is a 1.5 sample periods delay due to the DSP computational delay and the effect of the PWM. The fourth order approximation given in [12] is used to model this delay, so that an accurate representation is obtained at high frequencies, where the $L C L$ filter resonance is located.

Each block of the current control loop in Fig. 2, defined between brackets, is a $2 \times 2$ transfer function matrix in $d q$ axis. The transformation in [13] is used to rotate the transfer functions from their corresponding stationary frame to the synchronous frame. The angular speed at which each element is rotated is denoted by the subscript outside the brackets of each transfer function matrix. The elements of the GSC current control loop are defined in the stator stationary reference frame, $\alpha \beta_{s}$, thus, the rotation of these elements is made using $\omega_{0}$. In contrast, the elements of the RSC current control loop are defined in a stationary reference frame that rotates at the rotor electrical angular speed, $\omega_{m}$. This reference frame is denoted by $\alpha \beta_{m}$. In this case, the rotation of these elements to the synchronous reference frame is made with $\omega_{R}$. For this reason, there are different transfer function matrices for $L P A F(s)$ and $D_{\text {conv }}(s)$ in the GSC and the RSC.

As seen in Fig. 2, each converter has a different PI controller, $\left[P I_{G}\right]$ and $\left[P I_{R}\right]$, since their dynamics are different. These transfer function matrices are diagonal matrices since they are already defined in $d q$ axis. The expression of the diagonal terms is given by

$$
P I(s)=K_{p} \frac{T_{n} s+1}{T_{n} s}
$$

The variables that are measured in the current control loop must be transformed to the synchronous reference frame by means of the Park transformation. A phase-locked loop (PLL) is used for grid synchronization and it provides the angle for this transformation. Since the grid voltage cannot be measured, the filter capacitor voltage is used instead. Because of this, there is an interaction between the PLL and the current control loop that influences the dynamics of the system at low frequencies [14]. However, this paper focuses on analyzing the stability of the $L C L$ filter resonance, which occurs at high frequencies, therefore, the PLL is neglected in this model.

\section{Stability Analysis}

As a result of modeling the DFIG wind turbine in the synchronous reference frame using $2 \times 2$ transfer function matrices, a MIMO system is obtained. The stability of a MIMO system can be analyzed using the MIMO Generalized Bode Criterion (MIMO-GBC) [15], which is expressed as follows

$$
Z=P-\left[2\left(C^{+}-C^{-}\right)+C_{0}\right] .
$$

$Z$ is the number of closed-loop unstable poles and $P$ is the number of open-loop unstable poles. $C^{+}$is the number 
of $\pm m 180$ degree crossings ( $m$ odd integer) with positive magnitude and increasing phase at positive frequencies in the Bode diagram of all the system open-loop eigenvalues. $C^{-}$is the number of crossings with decreasing phase, and $C_{0}$ is the number of crossings at $0 \mathrm{~Hz}$.

The system stability is analyzed without applying any damping technique for the system parameters that appear in Table I, which are typical parameters of high power DFIG wind turbines. The slip is equal to -0.25 , with $s l i p=\frac{\omega_{R}}{\omega_{0}}$. First, we want to analyze the stability of each converter independently, controlling the complete plant, in order to determine whether, in this case, the instability of the DFIG system is generated by the interaction of both converters current control loops or it is inherent to each of them. For that purpose, the GSC and the RSC open-loop transfer function matrices, $\left[H_{G o l}\right]$ and $\left[H_{R o l}\right]$, are calculated imposing $V_{R d q}=0$ when computing [ $\left.H_{G o l}\right]$, and $V_{G d q}=0$ to obtain $\left[H_{R o l}\right]$. Fig. 3 shows the eigenvalues Bode diagram of $\left[H_{G o l}\right]$ and $\left[H_{R o l}\right]$ for a SCR of 10 . A SCR equal to 10 is chosen as an example to illustrate the stability analysis but the same results are obtained for any SCR. For the GSC eigenvalues there are two crossings at $0 \mathrm{~Hz}$, one in each eigenvalue, $C_{0}=-2$, and one crossing with 180 degrees with positive magnitude and increasing phase, $C^{+}=1$. There are also two \pm 180 degree crossings with decreasing phase, thus $C^{-}=2$. Since there are no open-loop unstable poles, according to (8) there must be 4 unstable closed-loop poles. In the case of the RSC, there are no crossings at $0 \mathrm{~Hz}$, nor crossings with increasing phase. However, there are also two \pm 180 degree crossings with decreasing phase so $C^{-}=2$. There are no open-loop unstable poles, therefore there are 4 unstable closed-loop poles as well. It is proved that both converters control loops are unstable separately.

However, in reality the GSC and the RSC are connected in parallel to the grid through the inductor $L_{G S C}$ and the DFIG, respectively. Therefore, there is an interaction between both

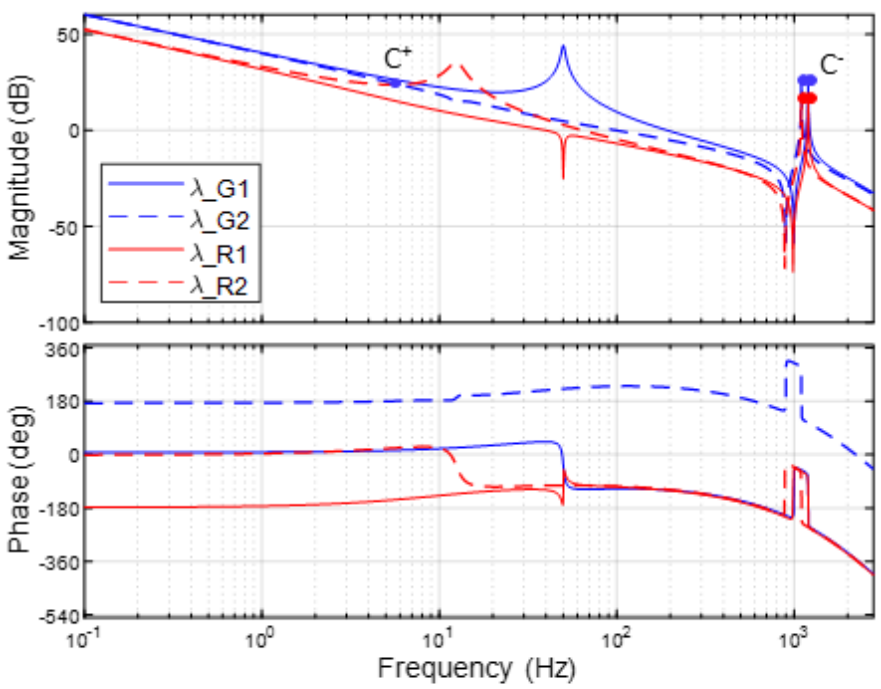

Fig. 3. Eigenvalues Bode diagram of the GSC and the RSC open-loop transfer matrix neglecting the interaction between both converters for a SCR of 10 .

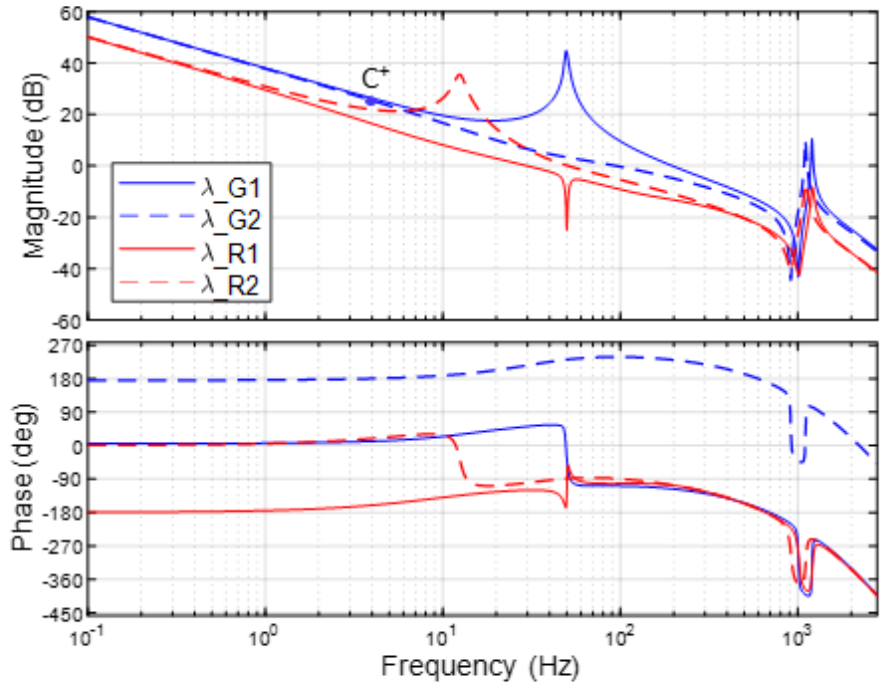

Fig. 4. Eigenvalues Bode diagram of the GSC and the RSC open-loop transfer matrix considering the interaction between both converters for a SCR of 10 .

control loops that must be considered in the stability analysis. For that purpose, the GSC open-loop transfer function matrix is calculated imposing $I_{R r e f d q}=0$. Similarly, $\left[H_{R o l}\right]$ is computed considering $I_{\text {Grefdq }}=0$. The eigenvalues Bode diagram for a SCR of 10 are represented in Fig. 4. For the GSC there is one crossing with 180 degrees with positive magnitude and increasing phase, $C^{+}=1$, which is compensated by two crossings at $0 \mathrm{~Hz}$, one in each eigenvalue, $C_{0}=-2$. However, there are 4 unstable open-loop poles that are introduced when closing the control loop of the other converter, the RSC, as seen in the previous stability analysis of each current control loop independently. Thus, the complete closed-loop system has four unstable poles, $Z=4$. The same result is obtained by analyzing the eigenvalues of $\left[H_{R o l}\right]$. There are no crossings with \pm 180 degrees but there are 4 open-

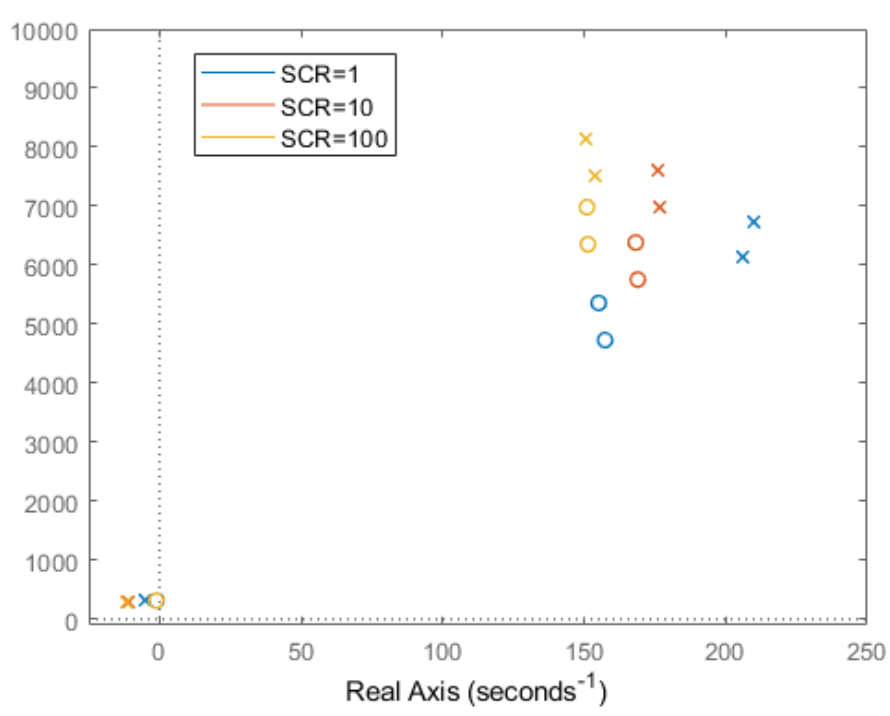

Fig. 5. Closed-loop poles of the system for three SCRs. 
loop unstable poles, introduced when closing the GSC control loop, that are translated to the closed-loop. Thus, the complete DFIG system is unstable. This can be verified by analyzing the closed-loop poles of the system represented in Fig. 5, for three SCRs that cover the whole range of possible resonance frequencies. In this figure only two poles appear because they are complex conjugate poles, so they are symmetric with respect to the real axis. It is proved that the system is unstable for any SCR. The frequency of the unstable poles corresponds to the equivalent resonance frequency of the $L C L$ filter in $d q$ coordinates. Therefore, it is shown that a damping technique is necessary to guarantee the stability of the system.

TABLE I

SYSTEM PARAMETERS

\begin{tabular}{|c|c|}
\hline Parameter & Magnitude \\
\hline \multicolumn{2}{|c|}{$\begin{array}{c}\text { DFIG } \\
S=5 M V A, U=690 \mathrm{~V}\end{array}$} \\
\hline Rotor resistance & 0.009 p.u. \\
\hline Rotor leakage inductance & 0.15 p.u. \\
\hline Stator resistance & 0.009 p.u. \\
\hline Stator leakage inductance & 0.111 p.u. \\
\hline Magnetizing inductance & 5.708 p.u. \\
\hline \multicolumn{2}{|c|}{ Power converter } \\
\hline Switching frequency & $2.8 \mathrm{kHz}$ \\
\hline Sampling frequency & $5.6 \mathrm{kHz}$ \\
\hline Converter side inductance & 0.284 p.u. \\
\hline Converter side resistance & 0.004 p.u. \\
\hline Filter capacitor & 0.021 p.u. \\
\hline Transformer inductance & 0.174 p.u. \\
\hline Transformer resistance & 0.015 p.u. \\
\hline \multicolumn{2}{|c|}{ Control parameters } \\
\hline GSC PI proportional gain & 0.1135 \\
\hline GSC PI integral constant & 0.0044 \\
\hline RSC PI proportional gain & 0.3504 \\
\hline RSC PI integral constant & 0.0156 \\
\hline Low-pass analog filter time constant & $114.10^{-6}$ \\
\hline
\end{tabular}

\section{Proposed Active DAmping Strategy}

In this paper the capacitor current feedback is used to actively damp the $L C L$ filter resonance. However, the phase in the feedback path is adjusted by means of a lag compensator to achieve a dominant resistive behavior in the emulated virtual impedance within the range of possible resonance frequencies.

$\left[H_{A D}\right]$, in red in Fig. 2, is the AD transfer function matrix, which is defined in the synchronous reference frame. It is a diagonal matrix, whose diagonal terms are denoted by $H_{A D 1}(s)$. The AD should only modify the dynamics of the system in the range of resonance frequencies, thus it is filtered. A low pass analog filter, modeled in $d q$ coordinates by $[L P A F]_{\omega_{0}}$, already exists in the AD feedback loop, therefore a high pass digital filter is added in $H_{A D 1}(s)$. The filter cutoff frequency is set at half of the lower limit of the system resonance frequency range, so that it properly attenuates the AD action at low frequencies, without affecting it within the resonance frequency range. If the real part of the emulated virtual impedance is negative within the range of possible resonance frequencies, the resonance will be amplified, destabilizing the system. Besides, the real part of the virtual impedance is the one that damps the resonant poles, whereas the imaginary part modifies the resonance frequency and even introduces new resonances. For that reason, in order to maximize the damping, an impedance where the resistive component is dominant, compared to the reactance, is needed in the resonance frequency range. This is achieved by adjusting the delay in the $\mathrm{AD}$ feedback loop with a lag compensator. $k_{G}$ and $k_{R}$ are the proportional gains for each converter. The expression of the $\mathrm{AD}$ transfer function, $H_{A D 1}(s)$, is given by

$$
H_{A D 1}(s)=H P D F(s) \operatorname{Lag}(s) .
$$

(10) is used to calculate the necessary phase lag that the lag compensator must introduce at the central resonance frequency, $f_{r c}$, so that the total phase lag of the inner $\mathrm{AD}$ loop at $f_{r c}$ equals $\pi . f_{r c}=\left(f_{r l}+f_{r h}\right) / 2$, where $f_{r l}$ and $f_{r h}$ are the lower and upper limits of the resonance frequency range. This way, a pure resistor is emulated at $f_{r c}$, ensuring a robust damping of the system resonant poles in the full range of resonance frequencies.

$$
\begin{aligned}
& \varphi\left(\operatorname{Lag}\left(j \omega_{r c}\right)\right)= \\
& =\pi-\varphi\left(H P D F\left(j \omega_{r c}\right) D_{c o n v 1}\left(j \omega_{r c}\right) L P A F_{1}\left(j \omega_{r c}\right)\right)
\end{aligned}
$$

In (10), $D_{\text {conv } 1}(s)$ and $L P A F_{1}(s)$ are the diagonal terms of the transfer function matrices that model the low pass analog filter and the digital control delay. The transfer function of a lag compensator is given by

$$
\operatorname{Lag}(s)=\frac{\frac{s}{z}+1}{\frac{s}{p}+1},
$$

where $|z|>|p|$. (12)-(14) are used to calculate the zero, $z$, and the pole, $p . \omega_{r c}=2 \pi f_{r c}$ is the frequency of the maximum phase lag, $\phi$, which, in this case, is equal to the desired phase lag calculated before using (10). The elements of the capacitor current feedback loop are the same for both converters, therefore the required phase lag is identical. In this case, $\phi=-60.1$ degrees at $f_{r c}=1094 \mathrm{~Hz}$.

$$
\begin{gathered}
b=\frac{1-\sin \phi}{1+\sin \phi} \\
p=\sqrt{\frac{\omega_{r c}^{2}}{b}} \\
z=b \cdot p
\end{gathered}
$$

Fig. 6 shows the cosine of the total phase lag of the inner $\mathrm{AD}$ loop, which is related to the real part of the virtual impedance that is emulated. When $H_{A D 1}(s)=1$ (dashed line) the lag is given by $D_{\text {conv } 1}(s)$ and $L P A F_{1}(s)$. After the AD function, $H_{A D 1}(s)$, is adjusted (continuous line), the real part of the virtual impedance is centered in the resonance frequency range, maximizing the damping in this interval. The emulated virtual resistor is negative with this strategy, thus the inner AD loop, represented in red in Fig. 2, becomes a capacitor current positive feedback. 


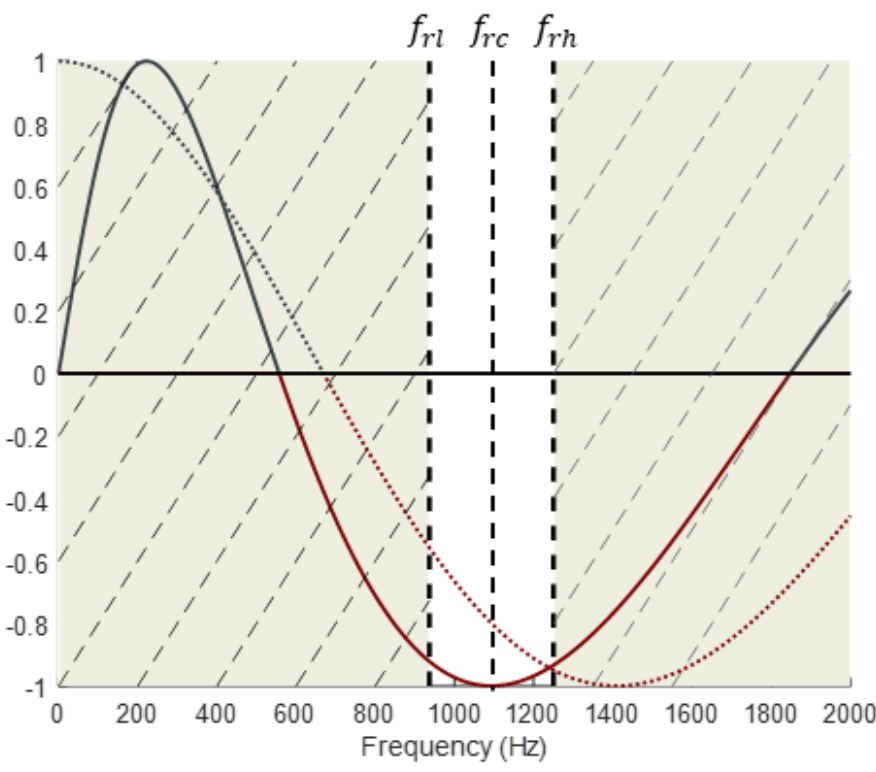

Fig. 6. Cosine of the phase lag of the inner AD loop against frequency before adjusting the delay (dashed line) and after (continuous line).

\section{A. Active Damping Implementation in the GSC}

First, the proposed active damping strategy is only implemented in the GSC. For that, the only element that needs to be adjusted is the proportional gain $k_{G}$. The closed-loop poles of the system are represented in Fig. 7 for different values of $k_{G}$ and a SCR of 10. The aim is to determine the optimal value of $k_{G}$ that provides the greatest damping to the system resonant poles. In this case, $k_{G}=1.4$, which results in the poles marked in red in the figure. As it is observed in Fig. 7, when the gain is too small (dark green poles), the resonant poles are not damped enough. As the gain increases, so does the damping. Nevertheless, once the optimal value has been reached, if the

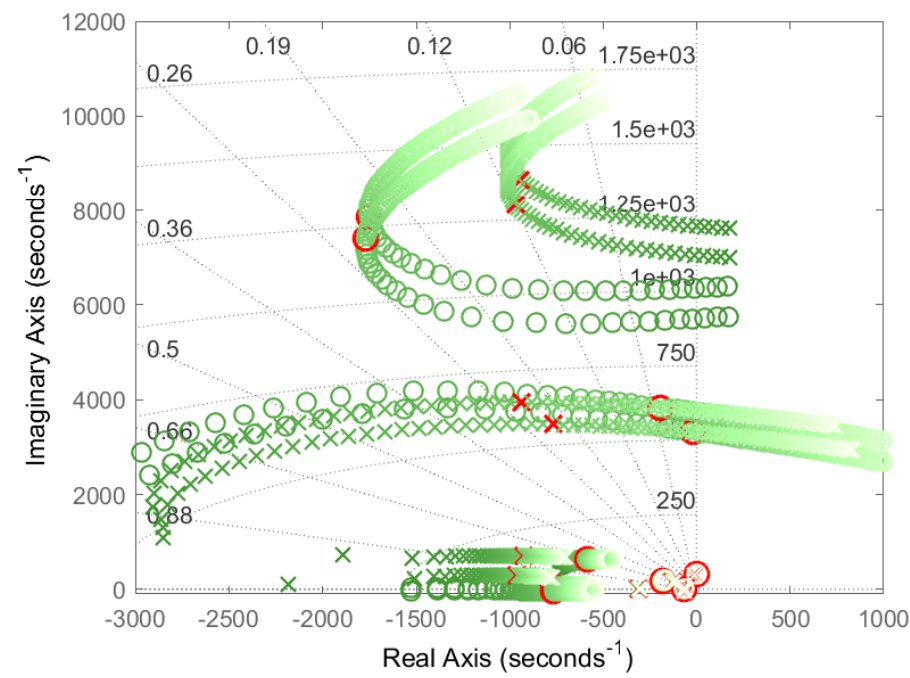

Fig. 7. Closed-loop poles of the system for different values of $k_{G}$ when the $\mathrm{AD}$ is only implemented in the GSC, for a SCR of 10 .

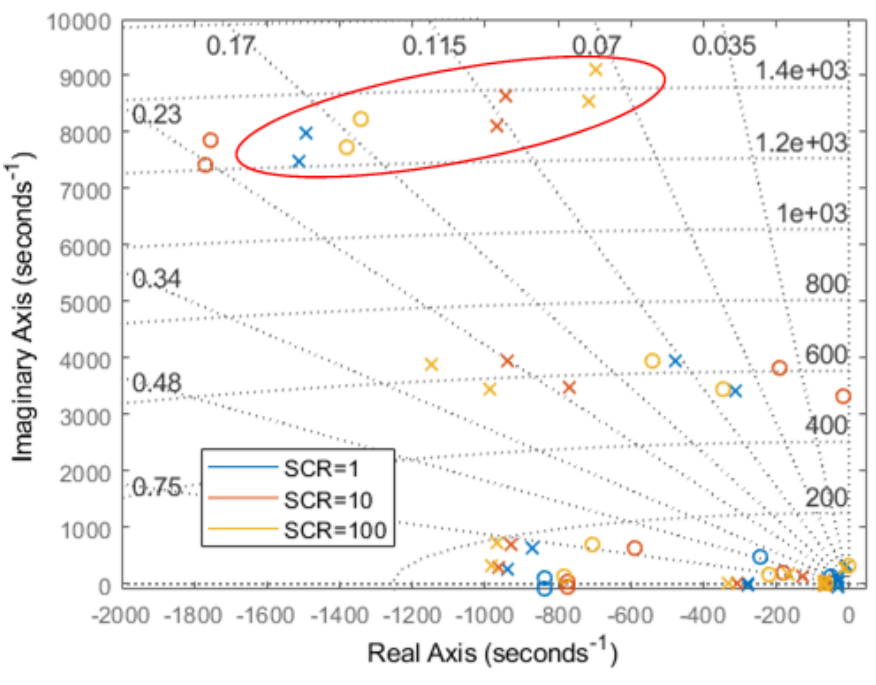

Fig. 8. Closed-loop poles of the system with the AD implemented in the GSC, for three SCR values.

gain continues to increase the system will eventually become unstable (light green poles).

The closed-loop poles of the DFIG system once the AD is adjusted for the GSC, are represented in Fig. 8, for three SCRs. It is observed that the proposed active damping strategy stabilizes the system resonant poles (circled in red) in a wide range of resonance frequencies, proving to be a robust strategy.

\section{B. Active Damping Implementation in the RSC}

A similar approach is followed to tune the proportional gain $k_{R}$ when the AD is only implemented in the RSC. By studying the system closed-loop poles, the optimal value of the gain that provides the greatest damping is determined, which is $k_{R}=4.1$. The closed-loop poles of the system, with the $\mathrm{AD}$ implemented in the RSC for three SCRs, are represented in

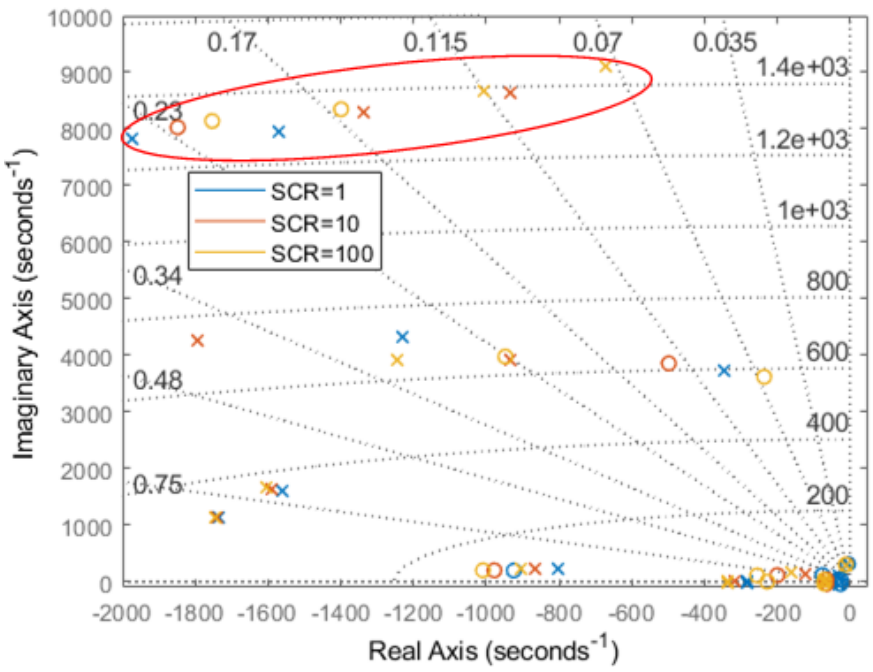

Fig. 9. Closed-loop poles of the system with the AD implemented in the RSC, for three SCR values. 


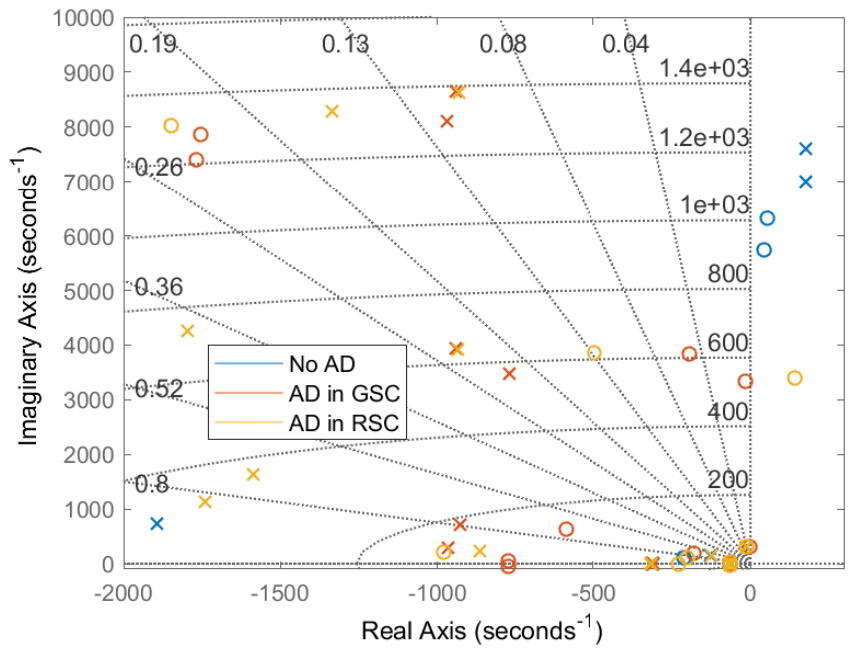

Fig. 10. Closed-loop poles of the system for three different control strategies for a SCR equal to 10 .

Fig. 9. As it is observed, the resonant poles, circled in red, are stabilized in all the cases, proving that the AD strategy is robust against grid impedance variations.

Fig. 10 shows the closed-loop poles of the system for three different control strategies: without $\mathrm{AD}$, with the proposed $\mathrm{AD}$ applied in the GSC and with the AD applied in the RSC, for a SCR of 10. Without an AD strategy the system is unstable, whereas the damping obtained with the other two strategies is $\xi=0.108$ and $\xi=0.107$, respectively. It is proved that the system can be damped by the implementation of the AD strategy in either of the two converters, obtaining a very similar damping of the resonant poles in both cases. The damping is not exactly the same because the plant seen from the GSC differs from the one seen from the RSC.

The inner AD loop in each converter modifies the system plant so that the complete DFIG system is stable, regardless of the converter in which the strategy is implemented. This way, the AD method can be applied in the converter that is more convenient, which is usually the one that is further from saturation.

\section{Simulation Results}

In order to validate the previous study, simulations are performed in Matlab. The Simscape Electrical Library is used to create a model of a DFIG wind turbine with the parameters that are reported in Table I. The control of each converter is programmed using two S-functions programmed in $\mathrm{C}$, and the modulation that is used is the space-vector PWM. The simulations are carried out for a grid SCR of 10 and a slip of -0.25 .

The grid current is shown in Fig. 11. At the beginning, the AD strategy is activated in the GSC and the system is stable. At $1 \mathrm{~s}$, the $\mathrm{AD}$ is disabled and the system becomes unstable. A component at the $L C L$ filter resonance frequency appears in the grid current. When the AD is then activated in the RSC, the system is stabilized again.

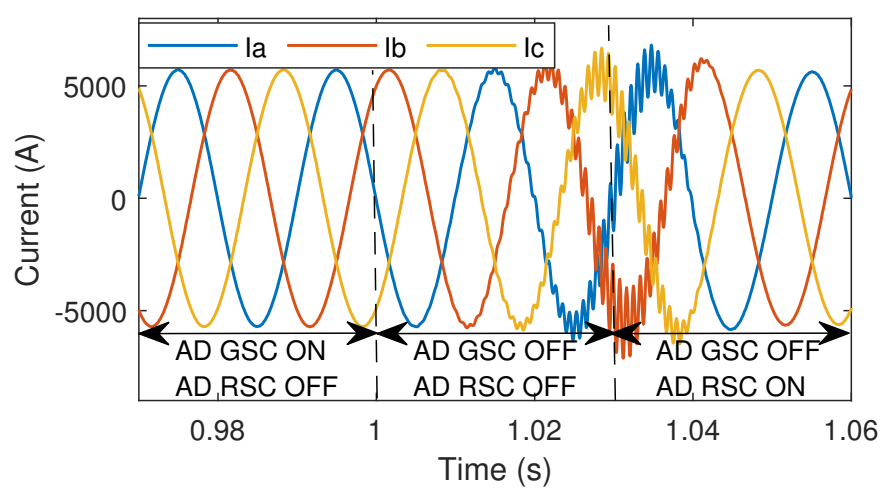

Fig. 11. Grid current waveform when the proposed AD strategy is activated and deactivated.

Fig. 12 shows the grid current harmonic content with the AD implemented in the GSC and in the RSC. The harmonic content is very similar using the two strategies as the system resonant poles are similarly damped in both cases. These simulation results validate the previous theoretical study, in particular the results from Fig. 10.

\section{CONCLUSION}

This paper proposes a robust AD strategy using the filter capacitor current for a DFIG wind turbine with $L C L$ filter. Despite the variety of $\mathrm{AD}$ strategies in the literature, they are not specific for DFIG systems, where the GSC and the RSC control loops, the DFIG and the $L C L$ filter interact with each other. In this paper this interaction is considered in the system model. A lag compensator is used in the AD feedback loop to adjust the delay so that the emulated virtual impedance is predominantly a resistance in the range of possible resonance frequencies, maximizing the damping in the full range. An

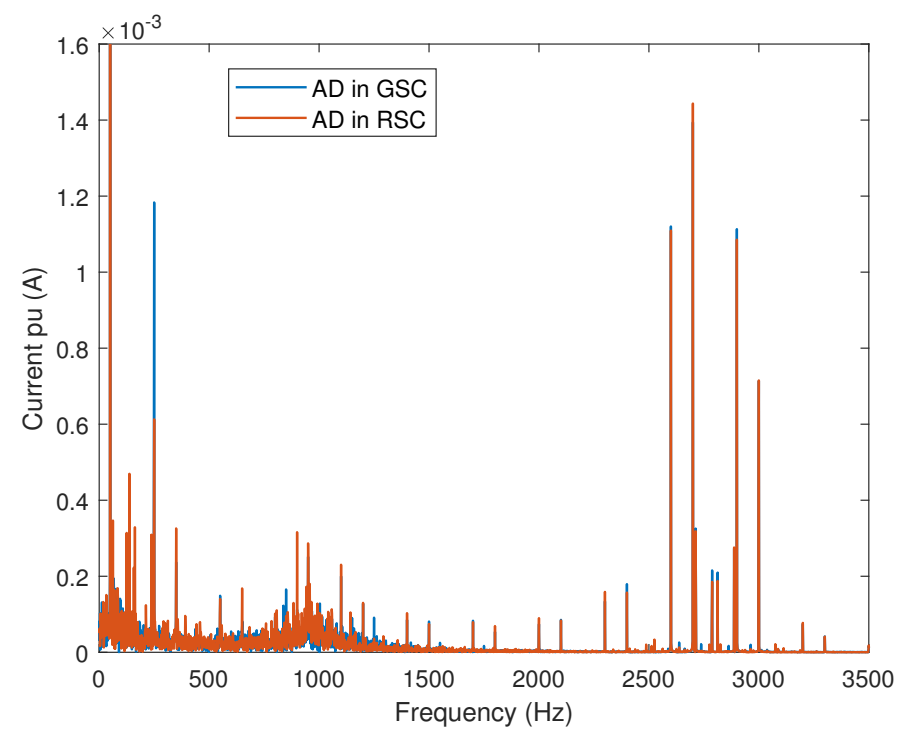

Fig. 12. Grid current harmonic content with the AD implemented in the GSC and in the RSC. 
equivalent damping in the resonant poles is achieved when the AD is applied in the GSC or the RSC, which offers more flexibility in the control strategy.

\section{REFERENCES}

[1] V. Yaramasu, B. Wu, P. C. Sen, S. Kouro, and M. Narimani, "Highpower wind energy conversion systems: State-of-the-art and emerging technologies," Proceedings of the IEEE, vol. 103, no. 5, pp. 740-788, 2015.

[2] M. Liserre, F. Blaabjerg, and S. Hansen, "Design and control of an lclfilter-based three-phase active rectifier," IEEE Transactions on industry applications, vol. 41, no. 5, pp. 1281-1291, 2005.

[3] W. Wu, Y. Liu, Y. He, H. S.-H. Chung, M. Liserre, and F. Blaabjerg, "Damping methods for resonances caused by lcl-filter-based currentcontrolled grid-tied power inverters: An overview," IEEE Transactions on Industrial Electronics, vol. 64, no. 9, pp. 7402-7413, 2017.

[4] J. Dannehl, M. Liserre, and F. W. Fuchs, "Filter-based active damping of voltage source converters with lcl filter," IEEE Transactions on Industrial Electronics, vol. 58, no. 8, pp. 3623-3633, 2010.

[5] W. Yao, Y. Yang, X. Zhang, F. Blaabjerg, and P. C. Loh, "Design and analysis of robust active damping for lcl filters using digital notch filters," IEEE Transactions on Power Electronics, vol. 32, no. 3, pp. 2360-2375, 2016.

[6] J. Samanes and E. Gubia, "Multisampled-capacitor-voltage active damping for parallel interleaved grid connected voltage source converters with 1cl filter," in 2017 19th European Conference on Power Electronics and Applications (EPE'17 ECCE Europe). IEEE, 2017, pp. P-1.

[7] D. Pan, X. Ruan, C. Bao, W. Li, and X. Wang, "Capacitor-currentfeedback active damping with reduced computation delay for improving robustness of lcl-type grid-connected inverter," IEEE Transactions on Power Electronics, vol. 29, no. 7, pp. 3414-3427, 2013.

[8] J. Liu, L. Zhou, and M. Molinas, "Damping region extension for digitally controlled lcl-type grid-connected inverter with capacitor-current feedback," IET Power Electronics, vol. 11, no. 12, pp. 1974-1982, 2018

[9] S. He, J. Xiong, Z. Wang, and S. Lin, "Robust ad for lcl-type gridconnected inverter with capacitor current quasi-integral feedback," IET Power Electronics, vol. 13, no. 7, pp. 1332-1342, 2020.

[10] Y. He, X. Wang, X. Ruan, D. Pan, X. Xu, and F. Liu, "Capacitor-current proportional-integral positive feedback active damping for lcl-type gridconnected inverter to achieve high robustness against grid impedance variation," IEEE Transactions on Power Electronics, vol. 34, no. 12, pp. 12 423-12 436, 2019.

[11] Y. Song, X. Wang, and F. Blaabjerg, "Impedance-based high-frequency resonance analysis of dfig system in weak grids," IEEE Transactions on Power Electronics, vol. 32, no. 5, pp. 3536-3548, 2016.

[12] J. Samanes and E. Gubía, "Sensorless active damping strategy for parallel interleaved voltage source power converters with lcl filter," in 2017 IEEE Applied Power Electronics Conference and Exposition (APEC). IEEE, 2017, pp. 3632-3639.

[13] D. N. Zmood, D. G. Holmes, and G. Bode, "Frequency domain analysis of three phase linear current regulators," in Conference Record of the 1999 IEEE Industry Applications Conference. Thirty-Forth IAS Annual Meeting (Cat. No. 99CH36370), vol. 2. IEEE, 1999, pp. 818-825.

[14] B. Wen, D. Boroyevich, R. Burgos, P. Mattavelli, and Z. Shen, "Analysis of dq small-signal impedance of grid-tied inverters," IEEE Transactions on Power Electronics, vol. 31, no. 1, pp. 675-687, 2015.

[15] J. Samanes, A. Urtasun, E. L. Barrios, D. Lumbreras, J. López, E. Gubia, and P. Sanchis, "Control design and stability analysis of power converters: The mimo generalized bode criterion," IEEE Journal of Emerging and Selected Topics in Power Electronics, vol. 8, no. 2, pp. 1880-1893, 2019. 